\title{
A High Brightness Electron Beam Produced by a Ferroelectric Cathode*
}

\author{
B. Jiang, G. Kirkman and N. Reinhardt \\ Integrated Applied Physics, Inc. \\ 50 Thayer Road, Waltham, MA 02154 USA \\ Tel. (617) 489-1818
}

\begin{abstract}
We report experimental measurements of a high brighuless electron beam produced by a ferroelectric cathode. The experiments were carried out with a cathode of area $3.7 \mathrm{~cm}^{2}$; up to $36 \mathrm{~A}$ of current $\left(10 \mathrm{~A} / \mathrm{cm}^{2}\right)$ were obtained at a voltage of $22.5 \mathrm{kV}$. The emission of the electron current was carried out with a DC voltage held between the cathode and ground anode. Emission was gated by a $1-2 \mathrm{kV}$, pulse applied across the sample by a solid state or krytron switch. The beam emittance was measured with a mask, phosphor screen and CCD camera; it was found to be $5 \pi \mathrm{mm}$ mrad yielding a normalized rms beam brightness of $1.2 \times 10^{11} \mathrm{~A} / \mathrm{m}^{2} \mathrm{rad}^{2}$. This is the first reported measurement of the brightness of a ferroelectric cathode beam; it exceeds that of a thermionic cathode and is close to that of a photocathodc. The results for the ferroelectric cathode may be directly compared with a preliminary experiment carried out in the same geometry with a thermionic cathode. The ferroelectric cathode yielded higher current density.
\end{abstract}

\section{Introduction}

Ferroelectric materials such as lead lanthanum zirconium titanate (PLZT) and lead titanate-zirconate (LTZ or PZT) become spontaneously polarized when cooled below their Curie temperature resulting in a high charge density at their surface which is normally screened by a layer of surface charge. The spontaneous polarization can be as high as $20 \mu \mathrm{C} / \mathrm{cm}^{2}$ resulting in the accumulation of a screening charge density of $10^{14}$ elementary charges $/ \mathrm{cm}^{2}$. Altering the spontaneous polarization rapidly results in emission of the surface charge layer. This emission mechanism is unique and has potential advantages over emission mechanisms presently considered for high brightness sources. Fast polarization change and electron emission are accomplished by applying a fast high voltage pulse to the ferroelectric disk. In our experiment, the electrodes are deposited silver coatings; one side is solid while the other is in a grid pattern leaving areas of the ferroelectric surface exposed. A 1-2 kV fast pulse is applied to the ferroelectric disk by a solid state or krytron switched pulse generator to change the polarization in the ferroelectric disk.

Electron emission from PLZT and PZT has been previously observed. Gundel and Riege [1], [2] of CERN

\footnotetext{
* This work was supported by National Science Foundation Grant No. ISI-9160797 through the SBIR Program.
}

obtained electron densities of $10 \mathrm{~A} / \mathrm{cm}^{2}$ and Ivers et al. [3] of Cornell obtained $70 \mathrm{~A} / \mathrm{cm}^{2}$. Both groups have measured electron beam current, current density, repetition rate capability and temporal evolution of the emission. In our work we have for the first time measured the quality of the beam produced by a ferroelectric cathode -..- emittance and brightness. We have obtained a beam current density similar to that of CERN and have shown that the beam is produced with low emittance.

\section{Experimental Design}

Electron emission is produced from LT7. ferroelectrics by rapidly changing the polarization of the ferroelectric with a fast rising high voltage pulse. The emitted electrons are then accelerated by an electron gun held at a DC potential by an external circuit. The ferroelectric electron gun consists of a ferroelectric disk with specially prepared contacts, an electron gun and two driving circuits -.- one to provide the fast high voltage pulse to induce the electron emission and the second to accelerate the beam in the electron gun. The electron gun and driving circuits are shown schematically in Figure 1

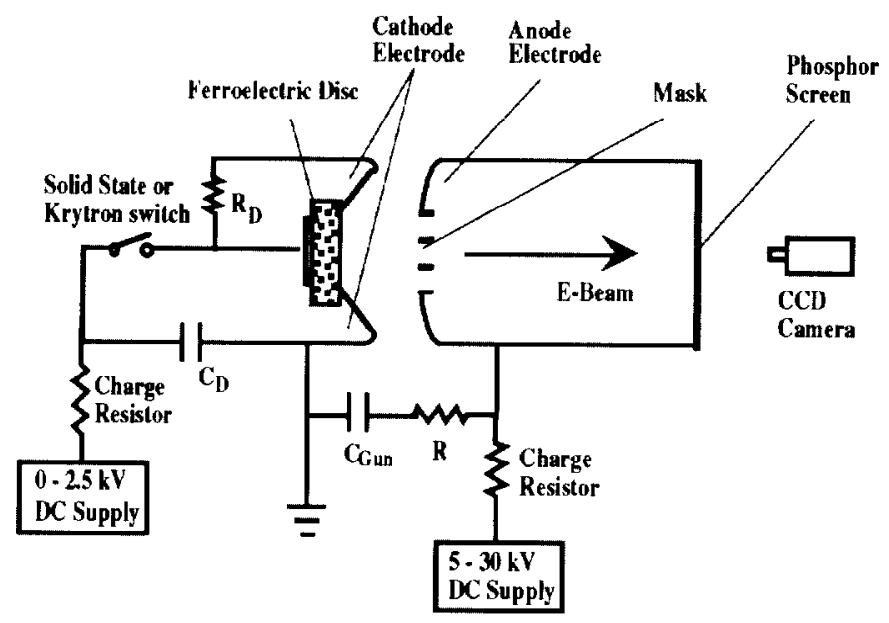

Figure 1. Electron gun and driving circuit for the ferroelectric experiment.

The driving circuit for inducing electron emission from the ferroelectric must produce a fast rising voltage pulse of 1 - $2 \mathrm{kV}$ across the ferroelectric disc. The ferroelectric disc has a capacitance of about $6 \mathrm{nF}$; therefore a high current pulser is required. Two tested pulsers are a commercial solid 
state switched pulser capable of producing $20 \mathrm{~A}$ at $2 \mathrm{kV}$ and a simple krytron switched pulser switching the charge stored in a $10 \mathrm{nF}$ capacitor to the ferroelectric

The electron source is a disc of LTZ-2 obtained from Transducer Products, Inc. The discs are $2.5 \mathrm{~cm}$ in diameter and $1 \mathrm{~mm}$ thick, with about 10 to $20 \mu \mathrm{m}$ thick silver contacts on both sides. The samples are pre-polarized in an electric field of $4 \mathrm{kV} / \mathrm{cm}$. To be used as an electron source the discs must have one contact in a grid pattern exposing the surface of the ferroelectric. At Integrated Applied Physics (IAP), we prepared this grid pattern by a photochemical etching process to give a pattern of $200 \mu \mathrm{m}$ wide silver strips separated by $200 \mu \mathrm{m}$ wide spaces of bare surface. A ring of solid silver material was left at the edge of the sample for electrical contact to the grid.

\section{Preliminary Experiments with a Thermionic Cathode}

The electron gun was designed to operate as a thermionic cathode at 5 - $50 \mathrm{kV}$ with space charge limited current for a flat thermionic cathode of a few $\mathrm{A} / \mathrm{cm}^{2}$. The space charge limited current of this gun was measured using the thermionic cathode to allow a direct comparison with the performance of the ferroelectric source. Results for the thermionic cathode are shown in Fig. 2. The gun has obtained its design perveance up to the limits of this measurement which are $40 \mathrm{kV}$ and $-3 \mathrm{~A} / \mathrm{cm}^{2}$ for a total current of $10 \mathrm{~A}$ and a perveance of $1.2 \mu$ Perv.

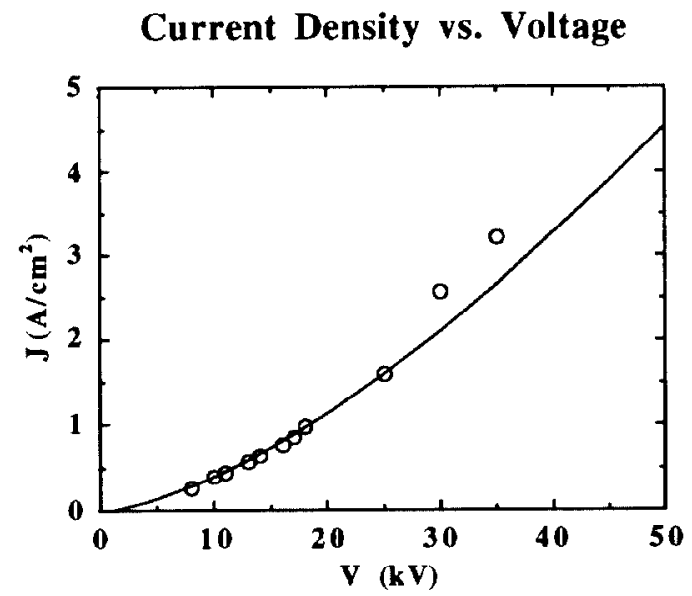

Figure 2. Electron gun results for the thermionic cathode. The curve is the theory for a $1.2 \mu$ Perv gun; the open circles are the experimental results. To obtain the electron beam current, multiply $\mathrm{J}$ by the cathode area, $3.7 \mathrm{~cm}^{2}$.

The ferroelectric electron gun was fabricated using high vacuum ceramic-metal techniques which we have developed for the fabrication of high power switches. All electrodes are stainless steel, the insulator is $\mathrm{Al}_{2} \mathrm{O}_{3}$ ceramic which is brazed to a Kovar ring, the entire assembly is welded together using TIG welding techniques developed at IAP. The final assembly is made using high vacuum flanges and the entire system is pumped on a diffusion pump to a base pressure of $10^{-7}$ Torr. For this work, we kept the electrode geometry of the thermionic electron gun, but replaced the thermionic cathode with the ferroelectric disk.

\section{Experimental Results for a Ferroelectric Cathode}

The measurements of the electron beam current versus applied gun voltage for the ferroelectric cathode are shown in Figure 3. The current increases linearly with the applied gun voltage and is higher than the result for a space charge limited thermionic cathode electron gun in the same geometry. The highest peak current observed was $36 \mathrm{~A}$ at a $22.5 \mathrm{kV}$ gun voltage, which is a current density of about 10 $\mathrm{A} / \mathrm{cm}^{2}$. The current observed is about 10 times larger than the expected space charge limited current for a thermionic cathode gun. At $15 \mathrm{kV}$ the ferroelectric source gives $24 \mathrm{~A}$ compared with the previously measured space charge limited current of $2.2 \mathrm{~A}$ at $15 \mathrm{kV}$ using a thermionic cathode. The results shown in Figure 3 qualitatively agree with the observations of the Comell group (Ref. 3). However, our peak beam current is lower than the expected circuit limit indicating that the electron gun or the ferroelectric source may be limiting the current.

Beam Current vs. Gun Voltage

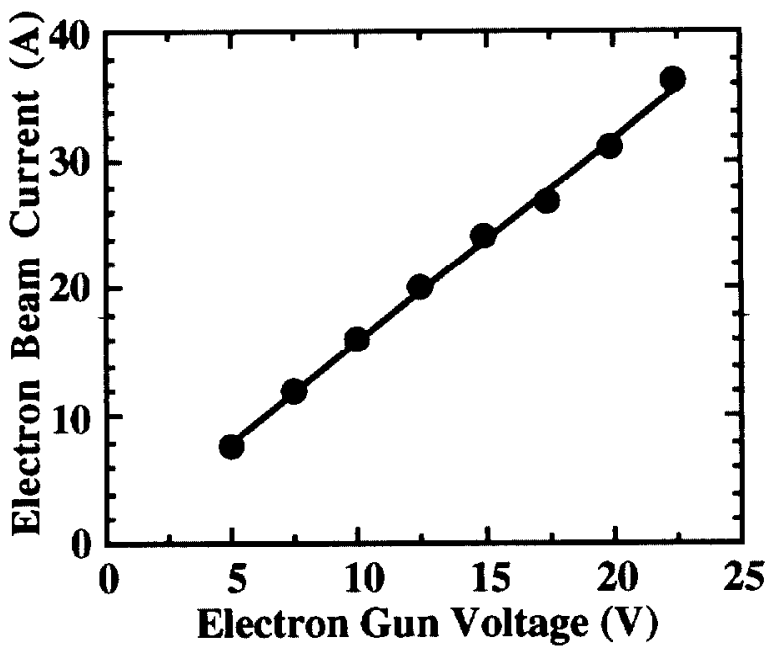

Fig. 3. Electron beam current produced versus applied gun voltage. In all cases the ferroelectric was pulsed at $2 \mathrm{kV}$.

Beam emittance has been measured by using the pepperpot emittance meter. In our implementation of this measurement, the pepperpot mask is a thin copper disk with an array of diameter $\mathrm{d}=200 \mu \mathrm{m}$ holes separated by $1 \mathrm{~mm}$. It is placed at the center of the anode electrode of the electron gun. A portion of the electron beam passes through the holes on the 
mask and drifts freely to a phosphor screen a distance $D=214$ $\mathrm{mm}$ away. The beam image on the phosphor screen is then recorded for analysis of the beam emittance. The beam is observed on the phosphor screen by using a CCD camera interfaced to a Macintosh computer. The image is captured and analyzed by using the program NIH Image 1.41. The combined resolution of the camera and the phosphor screen is better than $0.2 \mathrm{~mm}$ which allows us to measure beam emittance to about $\pm 10 \%$. The raw data of a density profile obtained by analyzing the beam image captured by the CCD camera are shown in Fig. 4.

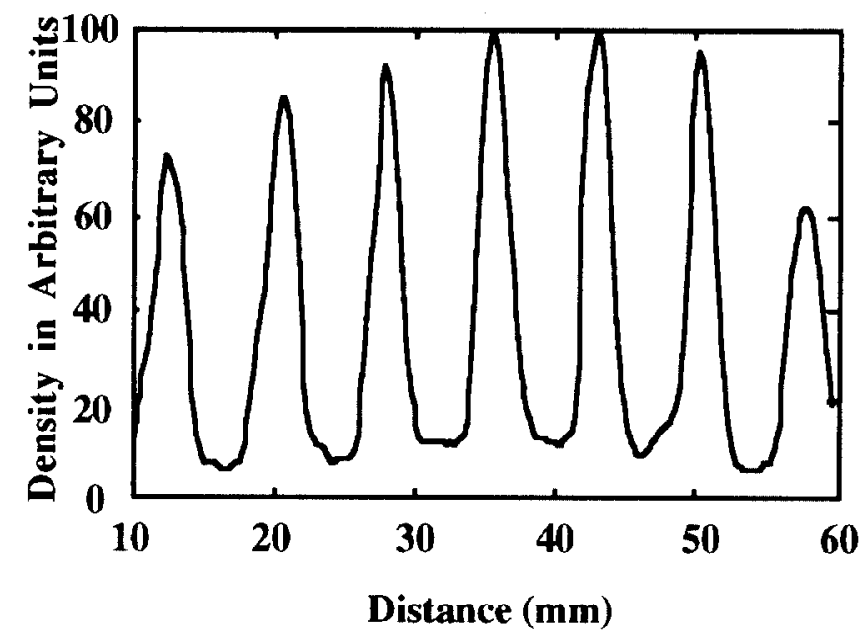

Fig. 4. Raw data of density profile.

Under the assumption that the electron beam produced by the ferrroelectric cathode is axisymmetric, not rotational and with a Maxwellian transverse velocity distribution, the data analysis approach of Rhee [4] is used to obtain the rms emittance of the beam. The normalized emittance at $10 \mathrm{kV}$ is $5 \pi \mathrm{mm} \cdot \mathrm{mrad}$. Emittance measurements were taken at several values of beam current between 3 and 15A. In this range, the emittance was observed to be constant. The normalized brightness at $10 \mathrm{kV}, 15 \mathrm{~A}$ is $1.2 \times 10^{11} \mathrm{~A} / \mathrm{m}^{2} \cdot \mathrm{rad}^{2}$.

\section{Discussion and Conclusions}

The present experiments have produced pulsed electron beams of very high quality from a novel, ferroelectric cathode. Operation was demonstrated at beam voltages of 5 to $22.5 \mathrm{kV}$ and currents up to $36 \mathrm{~A}$. At $10 \mathrm{kV}$ and $15 \mathrm{~A}$, the normalized emittance was measured to be $5 \pi \mathrm{mm} \mathrm{mrad}$ yielding a normalized beam brightness of $1.2 \times 10^{11} \mathrm{~A} / \mathrm{m}^{2} \mathrm{rad}^{2}$. These are, to our knowledge, the first reported measurements of the beam emittance and brightness of the electron beam produced by a ferroelectric cathode. A number of important applications appear promising for the ferroelectric cathode because of the demonstrated high beam quality.
The cathode was successfully prepared from a commercial specimen of LTZ-2 by etching a grid pattern. The ferroelectric disk was mounted in an electron gun geometry and sealed using high vacuum, ceramic-metal techniques. The final package is rugged and stable and should be capable of long life operation. The ability to demonstrate a rugged package for the novel ferroelectric cathode is another important factor in applying the cathode in research and commercial applications.

The ferroelectric cathode has many advantages over the conventional thermionic cathode which is widely used in microwave tubes, $x$-ray tubes, switches, etc. The present research gives direct experience in comparing the ferroelectric and thermionic cathodes in the same configuration. A thermionic cathode was able to produce about $3 \mathrm{~A}$ of current at $20 \mathrm{kV}$; in the same package, a ferroelectric cathode produced up to $30 \mathrm{~A}$ at $20 \mathrm{kV}$, an order of magnitude improvement. Furthermore, the quality of the ferroelectric beam was higher than that of the thermionic beam. The ferroelectric beam quality corresponded to an effective cathode temperature near room temperature; the effective temperature of the beam from a thermionic cathode is generally above $1000 \mathrm{C}$.

\section{References}

[1] H. Gundel H. Riege, E. Wilson, J. Handerek and K. Ziotus, Ferroelectrics 94, 337 (1989).

[2] H. Riege "New Ways of Electron Emission for Power Switching and Electron Beam Generation," in "Physics and Applications of Pseudosparks," NATO ASI Series B, Vol. 219, Edited by M. A. Gundersen and G. Schaefer, Plenum, New York, 1990.

[3] J. D. Ivers R. Advani, J. A. Nation and L Schachter, "Electron Emission from Ferroelectric Ceramics, 1991 Particle Accelerator Conference, San Francisco, CA, May, 1991.

[4] M J. Rhee and R. F. Schneider, "The Root-mean-square Emittance of an Axisymmetric beam with a Maxwellian Velocity Distribution", Particle Accelerators, 1986, Vol. 20 pp. 133 - 141.

\section{Acknowledgment}

The authors would like to thank Dr. R. J. Temkin for helpful suggestions during the course of this work. 